\title{
Stable structures of exohedrally decorated $\mathrm{C}_{60}$-fullerenes
}

\author{
Deb Sankar De ${ }^{\mathrm{a}, *}$, José A. Flores-Livas ${ }^{\mathrm{a}}$, Santanu Saha ${ }^{\mathrm{a}}$, Luigi Genovese ${ }^{\mathrm{b}}$, \\ Stefan Goedecker ${ }^{a}$ \\ ${ }^{a}$ Department of Physics, Universität Basel, Klingelbergstr. 82, 4056 Basel, Switzerland \\ ${ }^{b}$ Univ. Grenoble Alpes, CEA, INAC-MEM, L_Sim, F-38000, Grenoble, France
}

\begin{abstract}
A good hydrogen storage material should adsorb hydrogen in high concentrations and with optimal binding energies. Exohedrally metal decorated carbon fullerene structures were proposed as a promising material in this context. We present a fully $a b$-initio, unbiased structure search of the configurational space of decorated $\mathrm{C}_{60}$ fullerenes and find that many of the hitherto postulated ground state structures are not ground states. We determine the energetically lowest configurations for decorations with a varying number of decorating atoms $(2 \leqslant n \leqslant 32)$ for alkali metals, alkaline-earth metals as well as some other important elements and find that the dense uniform distribution of the decorating atoms over the surface of the $\mathrm{C}_{60}$, desired for hydrogen storage, can be obtained only for a few elements. An understanding of the behavior of the decorating atoms can be obtained by analyzing their bonding characteristics via the electron localization function.
\end{abstract}

Keywords: $\mathrm{C}_{60}$, Periodic Table, Exohedral, Wetting, Patching, ELF, Fullerenes

\section{Introduction}

Hydrogen-based technologies are a promising zero-carbon alternative to technologies using fossil fuels. However, the advancement of these technologies crucially depends on the availability of efficient hydrogen storage media [1, 2]. The

\footnotetext{
* Corresponding author

Email address: debsankar.de@unibas.ch (Deb Sankar De )
}

Preprint submitted to Elsevier

November 21, 2017 
mechanical approach relies on compressed gas storage, using advanced pressure vessels capable of sustaining high pressure at cryo-temperatures. The other approach searches for novel material-based hydrogen storage technologies. The materials in the later approach have to meet certain hydrogen storage target properties to be able to replace fossil fuels in practice $[3,1,4]$.

Certain decorated $\mathrm{C}_{60}$ fullerene structures have been assessed for its potential to meet these targets $[5,2,6,7,8]$. In particular, decorated fullerenes with alkali metals (AM) or alkaline-earth metals (AEM) enhances the hydrogen adsorption capacity $[2,7]$. The efficiency of a hydrogen storage material is measured by its gravimetric density (GD). For alkali metals the amount of hydrogen adsorbed on $\mathrm{C}_{60}$ increases with its coverage $[9,10]$. A maximum theoretical GD is 13 wt \% [9] for a $\mathrm{Li}_{12} \mathrm{C}_{60}$ cluster. The mechanism to stabilize hydrogen adsorption on fullerenes is the charge transfer from the metal atom to the fullerene cage, leaving the metal atom in a cationic state, which can bind $\mathrm{H}_{2}$ molecules due to polarization forces [11]. Experimentally, hydrogen storage for $\mathrm{Li}_{x} \mathrm{C}_{60}$ systems range between 5.0 to $5.9 \mathrm{wt} \%$ for bulk materials $[12,13,14,15]$.

It is clear that the geometry and type of coverage of the decorating atoms play a key role in determining the $\mathrm{H}_{2}$ adsorption mechanism. Pioneering work on this topic has been done by Martin and Zimmermann et al. $[16,17]$. They characterized the mechanism of atom decoration on $\mathrm{C}_{60}$ in the gas phase. In these early works, they found for lithium certain "magic numbers" which can only arise from an homogeneous distribution of twelve Li atoms on the top of the pentagonal faces of fullerene. Some other elements were found to form clusters above a certain concentration, or to form other non-homogeneous distributions on the surface $[18,19,20,21,22,23]$. This behavior is detrimental for hydrogen adsorption, because the clustering of the metal atoms on the surface of $\mathrm{C}_{60}$ reduces the active adsorption sites for $\mathrm{H}_{2}$ [24].

Because of the technical challenges of theoretical structure predictions, metal decorated $\mathrm{C}_{60}$ fullerenes were mainly studied experimentally. Also on the experimental level only a few decorating elements were studied due to the complexity of the experiments. Previous simulations were frequently performed using semi- 
empirical methods and hand-made geometries [25, 26]. Only a few attempts were made at the density-functional theory (DFT) level, but without fully exploring the potential energy landscape of decorated fullerenes [27, 28, 29, 30, 31, $32,33,34,7,6,35,36,37,38]$. A comprehensive and unbiased exploration of the potential energy surface at the $a b$ initio level requires special computational tools and methods that allow to calculate energies and ionic forces extremely rapidly [39]. Typically a simulation comprises between 60 to 100 atoms and the study of all possible configurations (hand made geometries) is not possible.

In this work we investigate exohedrally decorated atoms on $\mathrm{C}_{60}$ by employing for the first time a structure prediction method, namely minima hopping method (MHM) [40] at ab initio level. The energetically lowest configurations for exohedrally metal decorated $\mathrm{C}_{60} \mathrm{M}_{n}$ with $(2 \leqslant n \leqslant 32)$ is thus found for

alkali metals, alkaline-earth metals and some other elements. Furthermore, we can predict the behavior of a larger number of decorating atoms by analyzing the bonding characteristics (type and topology) of a single atom via the ELF (electron localization function).

\section{Methods}

The unbiased structure prediction of decorated fullerenes, fully at $a b$ initio level was conducted with the minima hopping method [40, 41, 42] . Given only the chemical composition of a system, the MHM aims at finding the global minimum on the potential energy surface while gradually exploring low-lying structures. The efficiency of the MHM method extensively depends on the exploitation of the Bell-Evans-Polanyi principle for molecular dynamics [43]. This method has been successfully used for global geometry optimization in a large variety of applications $[44,45,46,47,48,49,50,51,52,53]$. All the energy and force calculations were evaluated at the level of density-functional theory (DFT) using the Perdew-Burke-Ernzerhof exchange-correlation functional [54] as implemented in the wavelet basis based BIGDFT code [39]. The Libxc [55] library was used for the calculation of the functionals. We have employed soft dualspace Gaussian pseudopotentials with non-linear core correction [56, 57, 58, 59] 
and grid spacing of 0.4 Bohr along with tight electronic parameters such that the total energy difference are converged below $10^{-4} \mathrm{eV}$ for all stable configurations and geometry relaxation until forces on atoms are below $1 \mathrm{meV} / \AA$. The electron localization function (ELF) [60] was calculated on geometry relaxed structures using a plane wave basis-set with cutoff energy of $820 \mathrm{eV}$ within the projector augmented wave (PAW) method as implemented in the Vienna Ab Initio Simulation Package VASP [61] and in uniform boxes with size of $20 \times 20 \times 20 \AA$ for all the systems studies in this work. The adsorption energy is defined as:

$$
E_{\text {ads }}=E_{\mathrm{C}_{60}}+n \times E_{\text {atoms }}-E_{\text {Total }}
$$

Where $\mathrm{E}_{C_{60}}$ is the energy of $\mathrm{C}_{60}, \mathrm{E}_{\text {atoms }}$ is the energy of an atom, $n$ is the number of atom and $\mathrm{E}_{\text {Total }}$ is the total energy of the system. Here, the charge transfer is defined as the difference between the amount of electron present on an isolated atom and the total amount of charge present on the atom after the adsorption. This we obtained from the Bader charge analysis [62, 63, 64].

The ELF one-dimensional axis represents,

$$
\eta(r)=1 /\left(1+\left(D_{P} / D_{h}\right)^{2}\right)
$$

where $\mathrm{D}_{P}$ is the difference between the positive local kinetic energy of noninteracting electrons, having same density as the real system, and the von Weizsaecker kinetic energy functional and $\mathrm{D}_{h}$ is the local kinetic energy of a homogeneous electron gas. This function takes the values between 0 and 1. $\eta(r)=$ $1 / 2$ for instance represents a typical free-electron pairing. On the contrary, values close to 1 are typical of fully localized electron pairing. The important term $\mathrm{D}_{P}$ represents the excess local kinetic energy density due to the Pauli repulsion. Though the ELF has density-functional dependence, in this work we aim to capture first general trends over the periodic table treated within the same level of theory (GGA-PBE). 


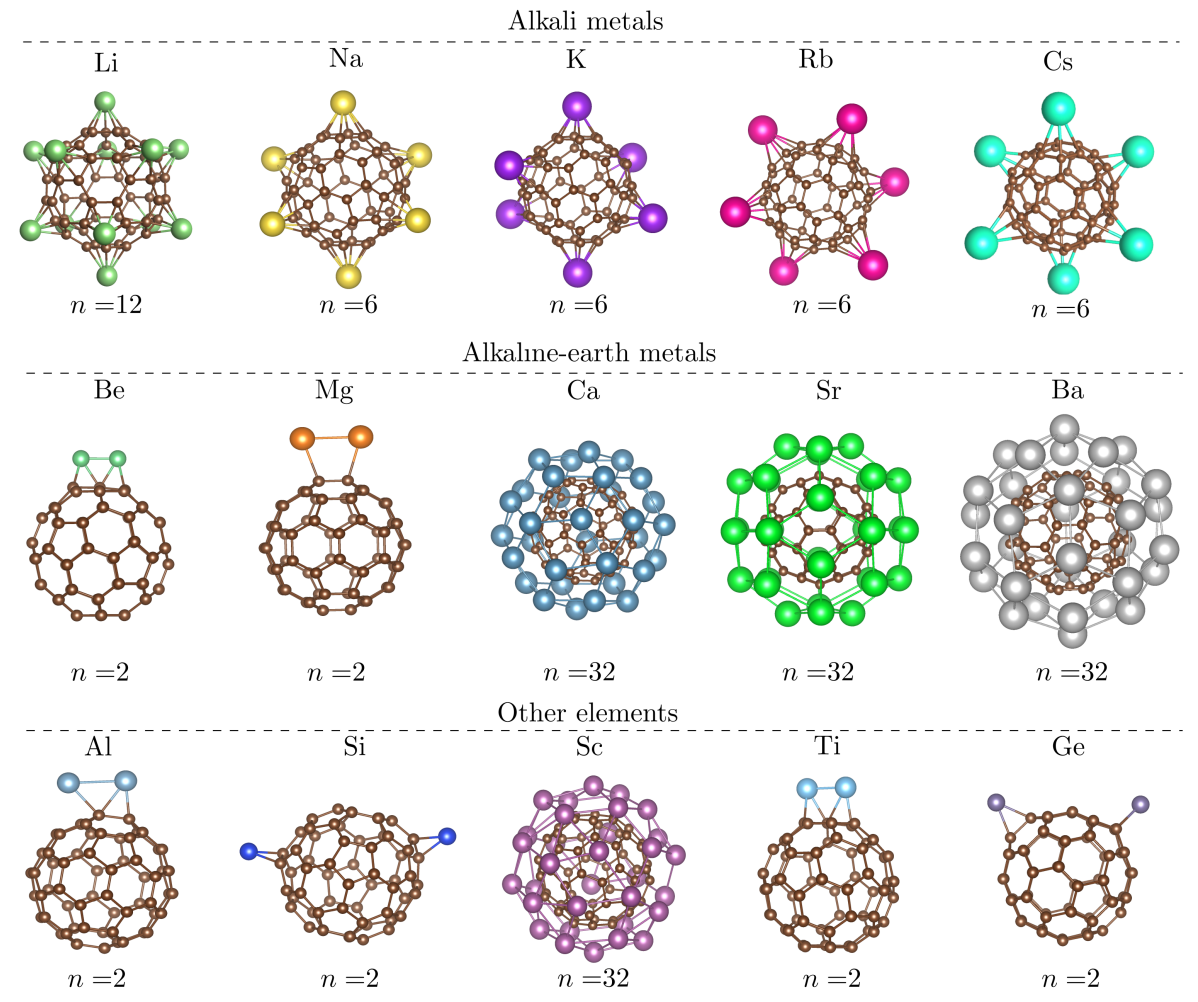

Figure 1: Maximum number of decorating atoms of the same species that can be homogeneously distributed on the $\mathrm{C}_{60}$ surface. For alkali metals a maximum of 12 atom is found for $\mathrm{Li}$, while for $\mathrm{Na}, \mathrm{K}$ and $\mathrm{Rb}$, it is reduced to 6 . For alkaline earth metals a maximum coverage of 32 atoms forming an outer-shell is achievable by $\mathrm{Ca}, \mathrm{Sr}$ and $\mathrm{Ba}$. The elements $\mathrm{Al}, \mathrm{Si}$. Ti and Ge in contrast do not form homogeneous decoration patterns. Sc (shown) and La (not shown) can form homogeneous distribution with up to 32 atoms.

\section{Results and discussion}

\subsection{Maximum exohedral decoration on $C_{60}$}

In the following we present the predicted stable configurations of the decorating atoms of different type on the surface of the fullerene. Three main scenarios have to be distinguished:

- The decorating atoms are uniformly distributed over the entire surface of the fullerene, maximizing their mutual distances. This behavior is know as wetting. 
Table 1: Summary of absorption energies $\left(\mathrm{E}_{a d s}\right)$, tendency for lone pair formation, ELF type and charge transfer for selected single atom-decorated $\mathrm{C}_{60} \mathrm{~s}$. The ELF type is determined through Fig. 5. $n$ is the maximum number of decorating atoms that can homogenously be distributed on a $\mathrm{C}_{60}$ as shown in Fig. 1. Non-homogeneous $(\mathrm{NH})$ distributions are also indicated. The ELF type for d)* systems is given in the supplementary information.

\begin{tabular}{c|c|c|c|c|c} 
Atom & $\begin{array}{c}\mathrm{E}_{a d s} \\
(\mathrm{eV})\end{array}$ & $\begin{array}{c}\text { Lone } \\
\text { pair }\end{array}$ & $\begin{array}{c}\text { ELF } \\
\text { type }\end{array}$ & $n$ & $\begin{array}{c}\text { Charge } \\
\text { transfer }\left(e^{-}\right)\end{array}$ \\
\hline $\mathrm{Li}$ & 1.79 & $\mathbf{x}$ & $\mathrm{b})$ & 12 & 1.00 \\
$\mathrm{Na}$ & 1.53 & $\mathbf{x}$ & $\mathrm{b})$ & 6 & 1.00 \\
$\mathrm{~K}$ & 1.88 & $\mathbf{x}$ & $\mathrm{b})$ & 6 & 1.00 \\
$\mathrm{Rb}$ & 1.59 & $\mathbf{x}$ & $\mathrm{b})$ & 6 & 1.00 \\
$\mathrm{Cs}$ & 1.70 & $\mathbf{x}$ & $\mathrm{b})$ & 6 & 1.00 \\
\hline $\mathrm{Be}$ & 0.29 & $\vee$ & $\mathrm{d})^{*}$ & $2(\mathrm{NH})$ & 1.50 \\
$\mathrm{Mg}$ & 0.07 & $\checkmark$ & $\mathrm{d})^{*}$ & $2(\mathrm{NH})$ & 0.12 \\
$\mathrm{Ca}$ & 1.37 & $\mathbf{x}$ & $\mathrm{c})$ & 32 & 1.42 \\
$\mathrm{Sr}$ & 1.03 & $\mathbf{x}$ & $\mathrm{c})$ & 32 & 1.48 \\
$\mathrm{Ba}$ & 1.85 & $\mathbf{x}$ & $\mathrm{c})$ & 32 & 1.40 \\
\hline $\mathrm{Sc}$ & 2.67 & $\mathbf{x}$ & $\mathrm{c})$ & 32 & 1.48 \\
$\mathrm{Y}$ & 2.38 & $\mathbf{x}$ & $\mathrm{c})$ & 32 & 1.59 \\
$\mathrm{La}$ & 3.37 & $\mathbf{x}$ & $\mathrm{c})$ & 32 & 1.50 \\
\hline $\mathrm{Zr}$ & 3.46 & $\mathbf{x}$ & $\mathrm{c})$ & 32 & 1.51 \\
$\mathrm{Ti}$ & 3.26 & $\checkmark$ & $\mathrm{d})^{*}$ & $2(\mathrm{NH})$ & 1.62 \\
$\mathrm{Si}$ & 2.77 & $\checkmark$ & $\mathrm{d})$ & 2 & 2.20 \\
$\mathrm{Ge}$ & 2.28 & $\checkmark$ & $\mathrm{d})$ & 2 & 1.80 \\
$\mathrm{Al}$ & 1.95 & $\checkmark$ & $\mathrm{d})^{*}$ & $2(\mathrm{NH})$ & 1.68 \\
& & & & &
\end{tabular}

- The decorating atoms strongly attract each other and form clusters, i.e. decorating atoms are clumped at a particular site on the surface with other atoms of the same species aggregated on top it. This will be called clustering.

- The decorating atoms form patches, i.e. a compact mono-layer covers a part of the surface of the fullerene. The atoms of same species never accommodate themselves on top of each other. This phenomenon is know as patching.

Fig. 1 summarizes the maximum number of atoms for all the elements considered that the surface of $\mathrm{C}_{60}$ can host before the system starts to show "clustering" or "patching". During the simulation of each system, several hundred configurations were visited. The MH runs are terminated when we observed 
that the energy of the system is significantly reduced, when the system starts to form clusters or when the basic structure of the fullerene is destroyed. Systems with a preference for homogeneously distributed configurations and highly symmetric patterns rapidly show an energy that is much lower than the energy of competing configurations.

First we studied the $\mathrm{C}_{60} \mathrm{M}_{n}$ structures for an increasing number $n$ of Li, $\mathrm{Na}$ and $\mathrm{K}$ atoms and observed that they wet $\mathrm{C}_{60}$ up to a limit of 12 atoms for $\mathrm{Li}$ and 6 for $\mathrm{Na}$ or $\mathrm{K}$ (Fig. 1). Rb/Cs atoms also wet $\mathrm{C}_{60}$ up to 6 atoms (Fig. 1) and for more number of atoms they start to form clusters. This is in agreement with previous experimental results [18]. $12 \mathrm{Sc}$ atoms do not wet as observed in previous theoretical results [35], but they form a patch. We scanned the PES using MHM and found out that the patched configuration is $10.66 \mathrm{eV}$ lower in energy then the homogeneously distributed configuration. As we increase the number of atoms, Ca and Sc also prefer to form patches. Ca and Sc can adsorb the largest number of atoms on the $\mathrm{C}_{60}$. They can homogeneously cover $\mathrm{C}_{60}$ with up to 32 atoms and form a core-shell type structure (Fig. 1). These findings are also in good agreement with the experimental evidences [18]. La also forms a homogeneous distribution, accommodating up to 32 atoms, making these new configurations interesting candidates for its synthesis.

If more than two atoms are present, $\mathrm{Si}$ and $\mathrm{Ge}$ starts to form cluster on the $\mathrm{C}_{60}$ surface. It is not surprising that silicon, germanium and carbon behave in a similar way and most other elements which form covalent bonds with carbon, show indeed a similar behavior. Our findings for Si and Ge are supported by experiment [22].

\subsection{Single atom on the fullerene surface}

To obtain further insights into the stability of decorated fullerenes, we searched the energetically lowest binding site for a single atom on the surface of the fullerene. We explored this for 64 elements. Fig. 2 shows the geometry optimized structures obtained with a single atom of $\mathrm{AM}, \mathrm{AEM}$ and $\mathrm{Al}, \mathrm{Si}, \mathrm{Sc}, \mathrm{Ti}$, and Ge. We found that the alkali metals (with the exception of Li) adopt the 

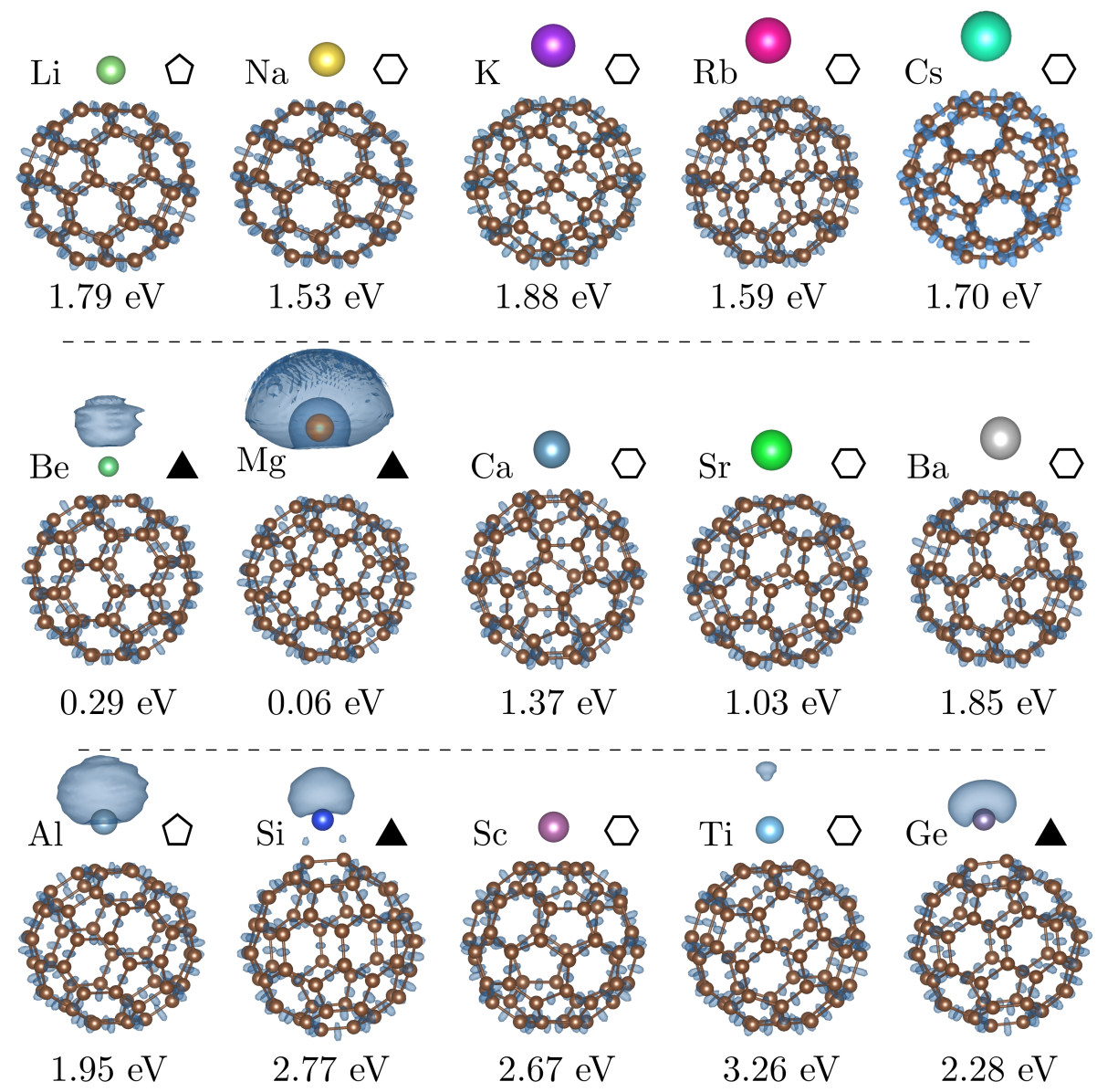

Figure 2: Different elements considered in this work: top row shows alkali metals, middle row alkaline-earth metals and bottom row other elements. The symbols next to the element symbol indicate the character of the energetically lowest adsorption site: (i) center of the hexagon (hexagon), (ii) center of the pentagon (pentagon) and (iii) On the C-C bond known as the "bridge site" (triangle). The calculated ELF at 0.9 for all the structures is shown for comparison (see text). For each structure the adsorption energy $\left(\mathrm{E}_{a d s}\right)$ is also given.

center of the hexagonal face as the lowest energy binding site. Lithium is the only element of this row with two degenerate sites. The calculated adsorption energy of a $\mathrm{Li}$ atom on the center of a pentagon or a hexagon site differs only by a few meV. A previous study reported very similar structures but a different energetic ranking. This is presumably due to different exchange-correlation functionals [27]. 
For the AEM family, only Be and Mg prefer to sit on the C-C bond ("bridge" site), whereas $\mathrm{Ca}, \mathrm{Sr}$ and $\mathrm{Ba}$ are most stable on the hexagonal face. Other elements considered in this study, which take the "bridge" site as the most stable site, are $\mathrm{Si}$ and Ge. Moreover, we found that a single $\mathrm{Al}$ atom prefers the pentagon site whereas a single Sc and Ti prefers the hexagonal site. The calculated electron localization function (ELF) at a value of 0.9 for all the systems is also shown Fig. 2.

Table 1 shows important properties for selected single atom-decorated $\mathrm{C}_{60}$. One can see some trends when comparing the adsorption site and the corresponding ELF (see adsorption values for each system). For instance, Be and $\mathrm{Mg}$ atom shows a lone pair electron and do not follow the behavior of the rest of their AEM family. Al, Si and Ge also display lone pair structures in their corresponding ELF. Our results for absorption energies $\left(\mathrm{E}_{a d s}\right)$ are in agreement with previous literature values. Especially for $\mathrm{Na}$ and $\mathrm{K}$ atoms the calculated adsorption energy is comparable to hybrid-functional (B3LYP) results [65, 66]. Single Be and $\mathrm{Mg}$ atoms occupy bridge-sites and are weakly adsorbed. The binding is weak due to the high ionization potentials of $\mathrm{Be}$ and $\mathrm{Mg}$ that hinders the donation of metal valence $s$ electrons to the fullerene. The adsorption energies for single atoms also correlate well with the ELF shown in Fig. 2. Higher adsorption energies imply higher stability and therefore lower total energy. Hence they are more likely to be accessible experimentally.

Fig. 3 shows the distance ( $y$-axis) between a single atom of a certain type and the nearest carbon atom of $C_{60}$ (green circles). The average C-C distance obtained after full geometry relaxation is also shown. It is interesting to observe some trends in the evolution of atom-carbon distance as function of the elements tested. The linear increase in distance between $\mathrm{Li}, \mathrm{Ca}, \mathrm{Sr}$ and $\mathrm{Ba}-\mathrm{C}$ can be explained by the increasing ionic radii for these elements. For other elements (Ti, Si, Ge, etc), which are strongly bound to the fullerene, the distance of interaction shrinks to $2.1 \AA$. The distance of Sc, Y and La, also correlates well with the increase of their ionic radii. Be and $\mathrm{Mg}$ have a relatively large metalcarbon distance. This is simply due to their weak bonding energies as described 


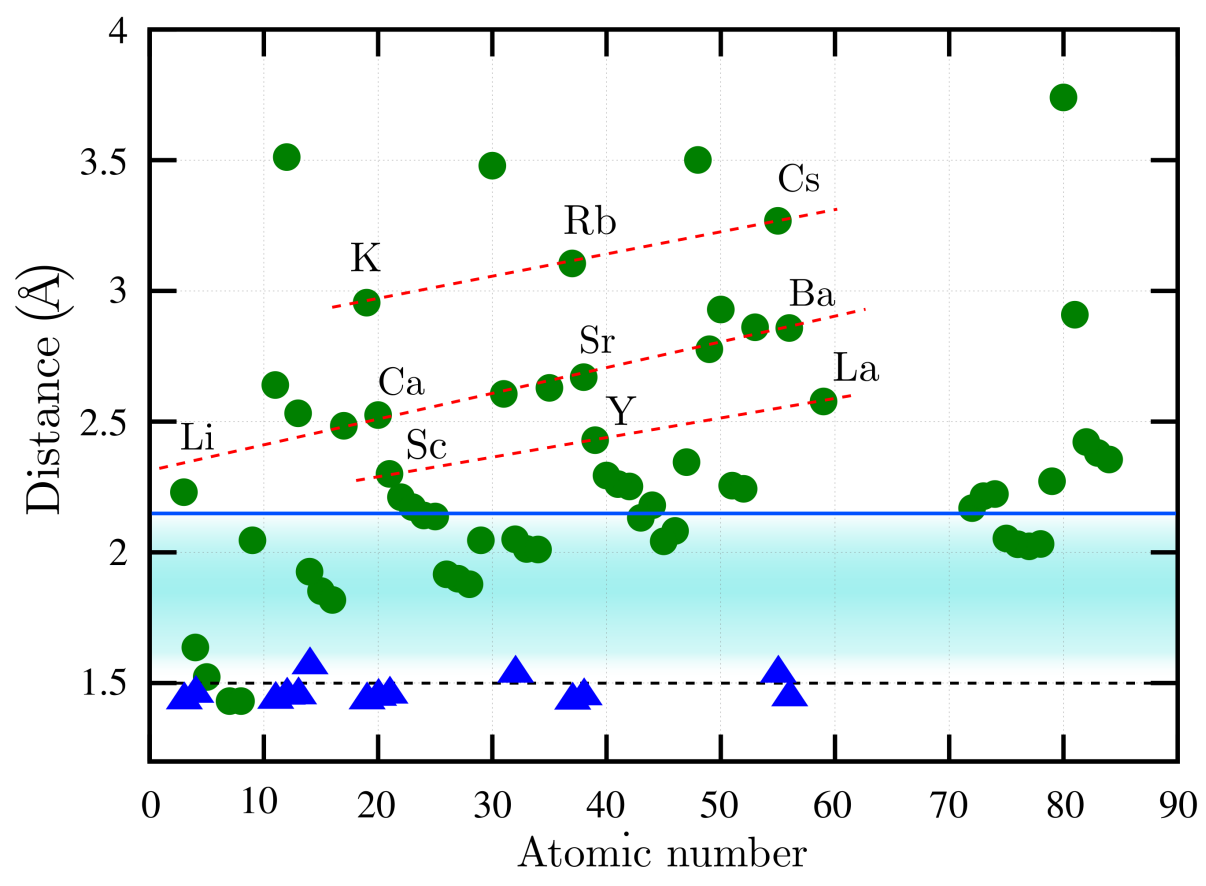

Figure 3: Trends in bond lengths for single atom decorating fullerenes. The green-circles represent the distance between the probe atom and the nearest carbon atom of the $\mathrm{C}_{60}$ for 64 elements sorted by atomic number. The respective average $\mathrm{C}-\mathrm{C}$ distance in $\mathrm{C}_{60}$ is shown for selected elements in blue-triangles. C-C distances do not deviate by more than $5 \%$ and remain thus close to $1.5 \AA$, the typical C-C distance in a isolated fullerene. Two major trends are: a linear increase in the bond lengths between the decorating atom and the closest carbon for $\mathrm{Li}$, $\mathrm{Ca}, \mathrm{Sr}$ and $\mathrm{Ba}$ and a constant length for $\mathrm{Be}, \mathrm{Si}$, and $\mathrm{Ge}$. Note that these same elements also form lone electron pairs (see ELF). The typical covalent bond between $\mathrm{C}$ and different metals in metallo-organic molecules lies in the range of $1.5 \AA$ to $2.2 \AA$ [67]. Here it is represented by the shaded area in between the black dotted line at $1.5 \AA$ to blue line line at $2.2 \AA$. Hence the elements which are homogeneously distributed over $\mathrm{C}_{60}$ have longer bond length than typical metallo-organic molecules.

before. The elements, with a interaction distance greater than $2.5 \AA$ ( $\mathrm{Na}, \mathrm{Mg}$, $\mathrm{Al}, \mathrm{K}$, and $\mathrm{Rb}$ ) have one unpaired electron and behave differently form the rest of the elements of the periodic table. It is noticeable that most of the $d$-block elements are found around the typical covalent distance lying between 2.0 to $2.4 \AA$.

\subsection{ELF analysis for decorated fullerene}

In the previous section, we have already given indication of the characteristic emerging for the different elements. More detailed information can be 


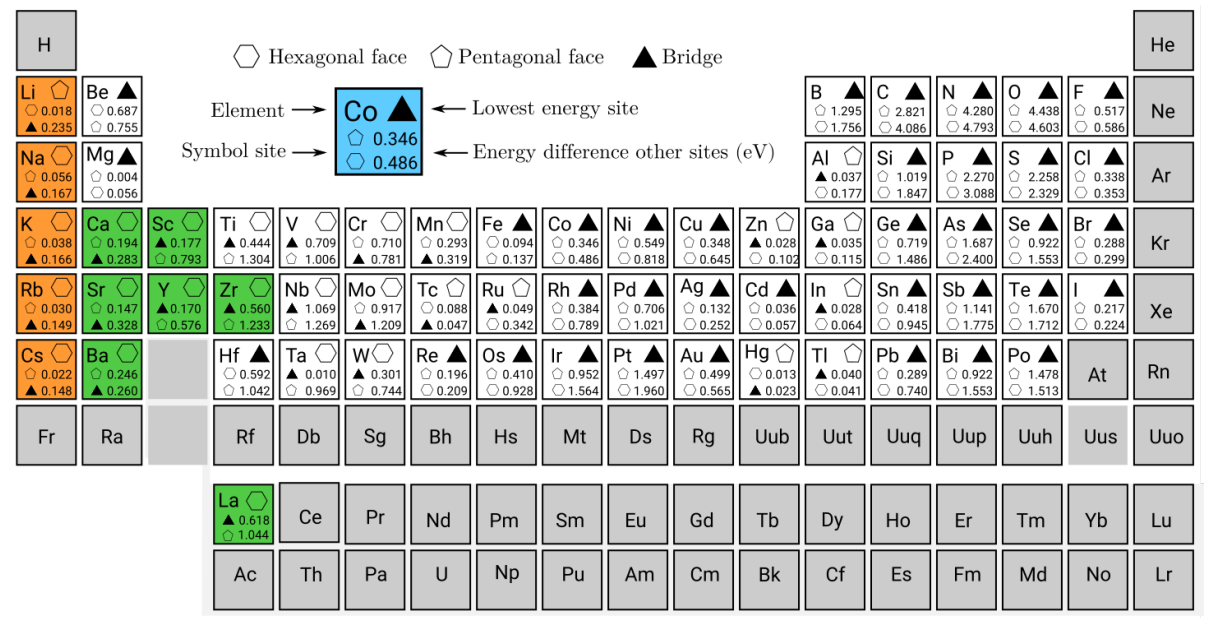

Figure 4: Periodic chart summarizing elements that are likely to form homogeneous decorated distributions and have a maximum coverage on the surface. 2 main types of electronic behaviors (ELF) are marked apart from the rest of the elements and those are depicted in orange and green, corresponding respectively to b) and c) in Fig. 5. For each atom, the energetically lowest site is indicated together with the difference in energy for other sites.

obtained by an ELF analysis. In particular the ELF analysis will allow us to predict whether the decorating atoms form a homogeneous distribution or undergo clustering or patching. (see methods for further details on ELF calculations).

Fig. 5 shows the different ELF volumes obtained for single atoms of different type on the surface of $\mathrm{C}_{60}$. ELF values $(\eta)$ vary from of 0.5 for free electrons to 1 for fully localized electrons. Values between $0.7-0.8$ indicate a covalent bond character. The analysis of the topology of the electronic structure by bifurcation hierarchies has been successfully used in many other systems $[68,69,70]$. Accordingly to their nature core and valence domains of bonding can be distinguished: core domain denoted as $\mathrm{C}(\mathrm{C})$ and $\mathrm{C}(\mathrm{M})$ for carbon and considered atom, respectively. Valence domains $\mathrm{V}(\mathrm{m})$ for atom on the surface or $\mathrm{V}(\mathrm{C})$ for carbon fullerene. A valence overlap composed of both the carbon and the considered atom domains, is denoted as $\mathrm{V}(\mathrm{C}, \mathrm{M})$.

The periodic chart of Fig.4 summarizes the results of the ELF analysis carried out for 64 different elements decorating the surface of $\mathrm{C}_{60}$. Despite the 

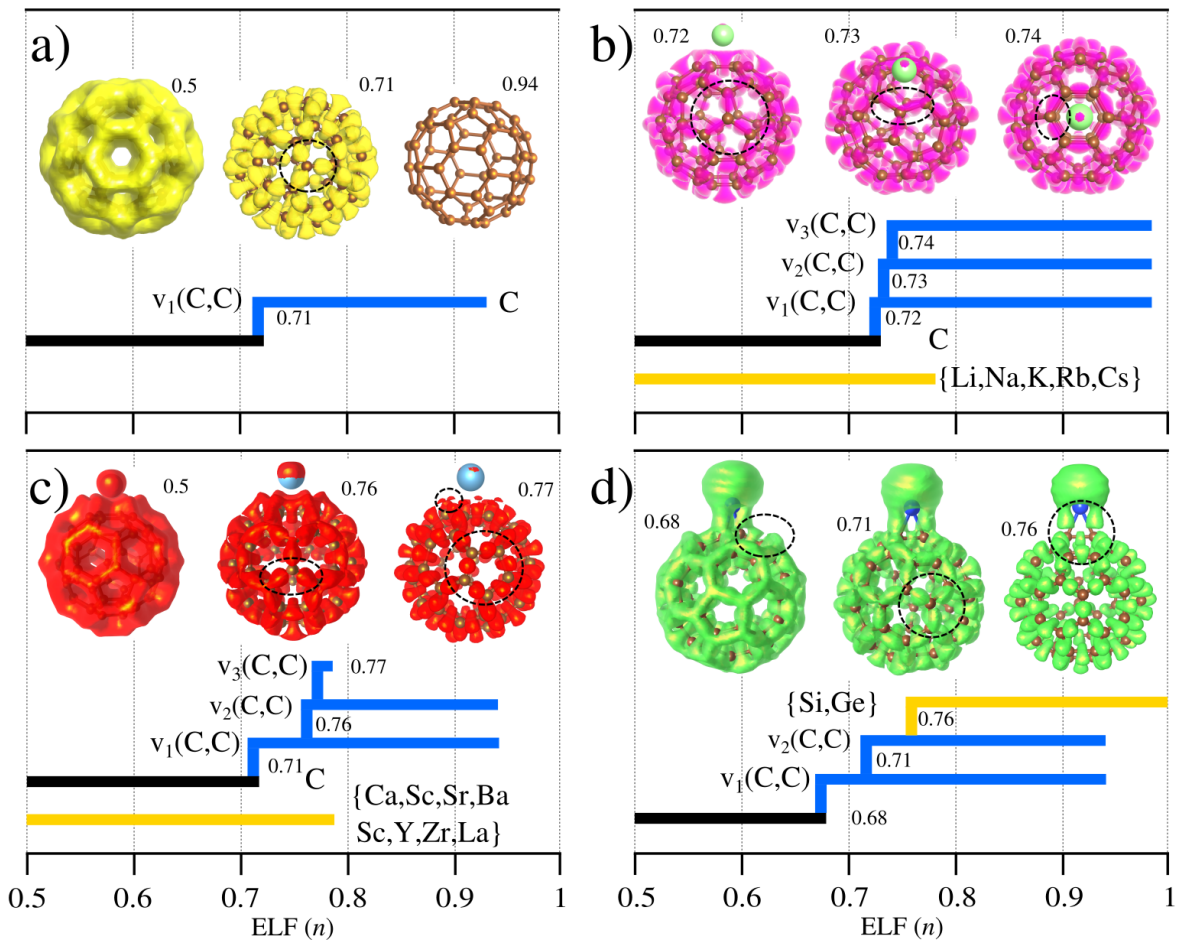

Figure 5: Electron localization function (ELF) analysis for different atoms: a) isolated $\mathrm{C}_{60}, \mathrm{~b}$ ) AM, c) AEM and d) for covalently bonded atoms. In these plots, black lines depicts the single basin (volume) and when it bifurcated, it is represented by blue lines. Yellow lines show the corresponding ELF of the atom considered. Clearly different levels of bifurcation (bonding) are distinguishable via the ELF. Other atoms that follow these patterns are indicated.

completely different nature of the 64 elements, a large number of atoms share a quite similar behavior and fall therefore in three classes that we will define. These classes are marked by different colors in Fig.4. For each atom, the energetically lowest site is indicated together with the difference in energy to other sites. The vast majority of elements will seat on "bridge" (indicated with solid triangles) sites forming covalent interactions. Only the orange and green color coded elements are predicted to form homogeneous decorated distributions that have a maximum coverage on the surface.

Both the ELF and Bader (not shown) analysis for single atoms on $\mathrm{C}_{60}$ reveal two distinct and general behaviors: (i) atoms with either covalent or ionic 
bonding do not show any lone pair electron and (ii) atoms that do form lone pairs electrons, weakly interact with the $\mathrm{C}_{60}$. From Fig. $2 \mathrm{i}$, we observe that the elements which have a lone pair (i.e. $\mathrm{Be}, \mathrm{Mg}, \mathrm{Al}$ ) also prefer to form clusters.

Let's analyze in more detail what is different for AM and AEM from the rest of elements. In all panels in Fig. 5 black solid line represents the main domain (continuous volume) and the blue lines are drawn only after a volume has bifurcated (discontinuous). Different bifurcations (blue lines) can be tracked and do correspond to the valence contributions $\left(\mathrm{V}_{1}, \mathrm{~V}_{2}\right.$ and $\left.\mathrm{V}_{3}\right)$. Yellow lines represent the atom probe on the surface of the fullerene. To further understand the bifurcation diagram, we should mention that the variation of $\eta$ is simply a measure of the number of the pairing electrons participating in the bonding (here depicted as the ELF volume). The variation of $\eta$ and consequently the volume variation, i.e. topology of the bonding, leading to either continuous or discontinuous volumes depending on the elf values, represent an reliable indicator for the behavior of a given decorating atom type. In this way ELF can be used to classify the different elements into two classes that share similar behavior as decorating atoms and remainder.

The first class comprises the alkali atoms $\mathrm{Li}, \mathrm{Na}, \mathrm{K}, \mathrm{Rb}$ and $\mathrm{Cs}$, (panel b. of Fig. 5 ). An elf analysis of the alkali elements suggests that they form strong ionic bonds with $\mathrm{C}_{60}$ [71]. The orange part in the periodic table (Fig. 4) represents this class of atoms. The ELF volume splits at $0.72,0.73$, and 0.74 values of $\eta$, representing three different valence electron contribution for $\mathrm{C}-\mathrm{C}$ bonds, respectively for $\left(\mathrm{V}_{1}(\mathrm{C}, \mathrm{C})\right),\left(\mathrm{V}_{2}(\mathrm{C}, \mathrm{C})\right)$, and $\left(\mathrm{V}_{3}(\mathrm{C}, \mathrm{C})\right)$. The first volume arises from the $\mathrm{C}-\mathrm{C}$ bonds which are far from the alkali atom. The second bifurcation is $\left(\mathrm{V}_{2}(\mathrm{C}, \mathrm{C})\right)$ which represents bonding between next nearest carbon atom and nearest carbon atom to the alkali atom. The valence basin $\left(\mathrm{V}_{3}(\mathrm{C}, \mathrm{C})\right)$ is induced by nearest carbon atoms and the alkali atom.

The second class comprises $\mathrm{Ca}, \mathrm{Sc}, \mathrm{Sr}, \mathrm{Ba}, \mathrm{Sc}, \mathrm{Y}, \mathrm{La}$ and $\mathrm{Zr}$ and is represented by green in the periodic table (Fig. 4). The ELF for these elements is characterized by three volume discontinuities at $\eta=0.71,0.76$ and 0.77 . This implies a similar bonding pattern as in the case of alkali atoms, except that for 
these metals, the third discontinuity is localized in space only for small $\eta$-values. This feature is visible in panel c) of Fig. 5 for a value of 0.77 (small volumes are indicated by a circle on the top of the fullerene).

The remaining elements shown in white in the periodic chart of Fig. 4 exhibit different ELF behavior but share the property of not homogeneously distributing over the $\mathrm{C}_{60}$ surface .

Most of the p-block elements have similar ELF behavior and up to two elements can be accommodated on the $\mathrm{C}_{60}$ surface before they start to form cluster.

We also include as reference the diagram for carbon, panel (a) in Fig. 5). The ELF analysis shows only one main discontinuity in the volume, occurring at 0.71 , which arises due to the purely covalent $\mathrm{C}-\mathrm{C}$ bonding in the isolated fullerene.

\section{Conclusions}

Fullerenes, exohedrally decorated with the alkali metals, alkaline-earth metals and other elements, were studied in a systematic, unbiased fashion with a structure search algorithm at the density-functional level. Our exploration of the potential energy surface resulted in new putative ground states and low energy configurations for a large number of elements and for a varying number of decorating atoms. We determined in particular the maximum number of atoms that can homogeneously be distributed on the fullerene surface. The ELF analysis helped to understand the behavior of AM and AEM and other elements when decorating fullerenes.

By scanning the ELF of 64 elements, we were able to determine: $\mathrm{Li}, \mathrm{Na}, \mathrm{K}$, $\mathrm{Rb}, \mathrm{Cs}, \mathrm{Ca}, \mathrm{Sr}, \mathrm{Ba}, \mathrm{Sc}, \mathrm{Y}$ and La as the sole elements that will favor homogeneous distributions. This picture explains the previous experimental findings for the elements $\mathrm{Li}, \mathrm{Na}, \mathrm{K}, \mathrm{Rb}, \mathrm{Cs}, \mathrm{Ca}, \mathrm{Sr}$ and $\mathrm{Ba}$. And for elements on which experimental results are absent, we predict that Y, Sc and La should also homogeneously cover $\mathrm{C}_{60}$. 


\section{Acknowledgments}

D.S.D and S.S acknowledge support from the Swiss National Science Foundation. D.S.D and J.A.F.L. acknowledge computing resources provided by the CSCS in Lugano under project s707 and s752. This work was done partially within the NCCR MARVEL project.

\section{References}

[1] L. Schlapbach, A. Züttel, Hydrogen-storage materials for mobile applications, Nature 414 (6861) (2001) 353-358.

[2] K. Chandrakumar, S. K. Ghosh, Alkali-metal-induced enhancement of hydrogen adsorption in $\mathrm{c}_{60}$ fullerene: an ab initio study, Nano letters 8 (1) (2008) 13-19.

[3] L. Pan, M. B. Sander, X. Huang, J. Li, M. Smith, E. Bittner, B. Bockrath, J. K. Johnson, Microporous metal organic materials: promising candidates as sorbents for hydrogen storage, Journal of the American Chemical Society 126 (5) (2004) 1308-1309.

[4] B. Bogdanović, M. Schwickardi, Ti-doped alkali metal aluminium hydrides as potential novel reversible hydrogen storage materials, Journal of alloys and compounds 253 (1997) 1-9.

[5] T. Yildirim, J. Íñiguez, S. Ciraci, Molecular and dissociative adsorption of multiple hydrogen molecules on transition metal decorated $\mathrm{c}_{60}$, Physical Review B 72 (15) (2005) 153403.

[6] Q. Wang, Q. Sun, P. Jena, Y. Kawazoe, Theoretical study of hydrogen storage in ca-coated fullerenes, Journal of Chemical Theory and Computation 5 (2) (2009) 374-379.

[7] M. Yoon, S. Yang, C. Hicke, E. Wang, D. Geohegan, Z. Zhang, Calcium as the superior coating metal in functionalization of carbon fullerenes for high-capacity hydrogen storage, Phys. Rev. Lett. 100 (2008) 206806. 
[8] Q. Wang, Q. Sun, P. Jena, Y. Kawazoe, Theoretical study of hydrogen storage in ca-coated fullerenes, Journal of chemical theory and computation 5 (2) (2009) 374-379.

[9] Q. Sun, P. Jena, Q. Wang, M. Marquez, First-principles study of hydrogen storage on li12c60, Journal of the American Chemical Society 128 (30) (2006) 9741-9745.

[10] Q. Wang, P. Jena, Density functional theory study of the interaction of hydrogen with li6c60, The journal of physical chemistry letters 3 (9) (2012) 1084-1088.

[11] B. Rao, P. Jena, Hydrogen uptake by an alkali metal ion, EPL (Europhysics Letters) 20 (4) (1992) 307.

[12] P. Mauron, M. Gaboardi, A. Remhof, A. Bliersbach, D. Sheptyakov, M. Aramini, G. Vlahopoulou, F. Giglio, D. Pontiroli, M. Ricco, et al., Hydrogen sorption in li12c60, The Journal of Physical Chemistry C 117 (44) (2013) 22598-22602.

[13] M. Gaboardi, N. S. Amadé, M. Aramini, C. Milanese, G. Magnani, S. Sanna, M. Riccò, D. Pontiroli, Extending the hydrogen storage limit in fullerene, Carbon 120 (2017) 77-82.

[14] L. Maidich, D. Pontiroli, M. Gaboardi, S. Lenti, G. Magnani, G. Riva, P. Carretta, C. Milanese, A. Marini, M. Ricco, et al., Investigation of li and h dynamics in li 6 c 60 and li 6 c 60 h y, Carbon 96 (2016) 276-284.

[15] M. Gaboardi, S. Duyker, C. Milanese, G. Magnani, V. K. Peterson, D. Pontiroli, N. Sharma, R. Mauro, In situ neutron powder diffraction of li6c60 for hydrogen storage, The Journal of Physical Chemistry C 119 (34) (2015) 19715-19721.

[16] T. Martin, N. Malinowski, U. Zimmermann, U. Näher, H. Schaber, Metal coated fullerene molecules and clusters, The Journal of chemical physics 99 (5) (1993) 4210-4212. 
[17] U. Zimmermann, A. Burkhardt, N. Malinowski, U. Näher, T. Martin, Quantum chemical study of lithium- ${ }^{60}$ clusters, The Journal of chemical physics 101 (3) (1994) 2244-2249.

[18] U. Zimmermann, N. Malinowski, A. Burkhardt, T. Martin, Metal-coated fullerenes, Carbon 33 (7) (1995) 995-1006.

[19] B. Palpant, A. Otake, F. Hayakawa, Y. Negishi, G. H. Lee, A. Nakajima, K. Kaya, Photoelectron spectroscopy of sodium-coated $c_{60}$ and $c_{70}$ cluster anions, Phys. Rev. B 60 (1999) 4509-4512.

[20] P. Dugourd, R. Antoine, D. Rayane, I. Compagnon, M. Broyer, Enhanced electric polarizability in metal $\mathrm{c}_{60}$ compounds: Formation of a sodium droplet on $c_{60}$, The Journal of Chemical Physics 114 (5) (2001) 1970-1973.

[21] Pellarin, M., Cottancin, E., Lermé, J., Vialle, J. L., Broyer, M., Tournus, F., Masenelli, B., Mélinon, P., Photodissociation and photoionization of sodium coated $c_{60}$ clusters, Eur. Phys. J. D 25 (1) (2003) 31-40.

[22] M. Ohara, Y. Nakamura, Y. Negishi, K. Miyajima, A. Nakajima, K. Kaya, Behavior of silicon and germanium clusters on a $c_{60}$ fullerene, The Journal of Physical Chemistry A 106 (18) (2002) 4498-4501.

[23] M. Harnisch, M. Daxner, P. Scheier, O. Echt, Adsorption of sodium and cesium on aggregates of $\mathrm{c}_{60}$, The European Physical Journal D 70 (9) (2016) 192.

[24] Q. Sun, Q. Wang, P. Jena, Y. Kawazoe, Clustering of ti on a $c_{60}$ surface and its effect on hydrogen storage, Journal of the American Chemical Society 127 (42) (2005) 14582-14583.

[25] H. Tanaka, S. Osawa, J. Onoe, K. Takeuchi, Formation process of si-coated $\mathrm{c}_{60}$, The Journal of Physical Chemistry B 103 (29) (1999) 5939-5942.

[26] F. Rabilloud, R. Antoine, M. Broyer, I. Compagnon, P. Dugourd, D. Rayane, F. Calvo, F. Spiegelman, Electric dipoles and susceptibilities 
of alkali clusters/fullerene complexes: experiments and simulations, The Journal of Physical Chemistry C 111 (48) (2007) 17795-17803.

[27] N. Hamamoto, J. Jitsukawa, C. Satoko, Electronic and geometric properties of alkali-c $\mathrm{c}_{60}$ molecules, The European Physical Journal D-Atomic, Molecular, Optical and Plasma Physics 19 (2) (2002) 211-221.

[28] J. Roques, F. Calvo, F. Spiegelman, C. Mijoule, Wetting-to-nonwetting transition in metal-coated $c_{60}$, Phys. Rev. Lett. 90 (2003) 075505.

[29] J. Roques, F. Calvo, F. Spiegelman, C. Mijoule, Nucleation of a sodium droplet on $\mathrm{c}_{60}$, Phys. Rev. B 68 (2003) 205412.

[30] F. Rabilloud, R. Antoine, M. Broyer, I. Compagnon, P. Dugourd, D. Rayane, F. Calvo, F. Spiegelman, Electric dipoles and susceptibilities of alkali clusters/fullerene complexes âĂL' experiments and simulations, The Journal of Physical Chemistry C 111 (48) (2007) 17795-17803.

[31] P. Weis, R. D. Beck, G. Bräuchle, M. M. Kappes, Properties of size and composition selected gas phase alkali fulleride clusters, The Journal of chemical physics 100 (8) (1994) 5684-5695.

[32] A. Tang, Z. Shang, Q. Teng, Y. Pan, Z. Cai, X. Zhao, J. Feng, Theoretical study on the structures and electron spectra of $c_{60} \mathrm{~m}_{12}(\mathrm{~m}=\mathrm{li}$, na, be), International journal of quantum chemistry 73 (6) (1999) 505-509.

[33] Z. Slanina, C. Miyajima, X. Zhao, F. Uhlík, L. Adamowicz, E. sawa, Metalcoated fullerenes $\mathrm{c}_{60} \mathrm{~m}_{n}$ : Calculations for $\mathrm{m}=\mathrm{be}, \mathrm{mg}$, al and $\mathrm{n}=12,20$, 32, Fullerene science and technology 8 (4-5) (2000) 385-402.

[34] H. Lee, B. Huang, W. Duan, J. Ihm, Ab initio study of beryllium-decorated fullerenes for hydrogen storage, Journal of Applied Physics 107 (8).

[35] Y. Zhao, Y.-H. Kim, A. Dillon, M. Heben, S. Zhang, Hydrogen storage in novel organometallic buckyballs, Physical review letters 94 (15) (2005) 155504 . 
[36] R. Guirado-López, Stability and electronic properties of si-doped carbon fullerenes, Phys. Rev. B 65 (2002) 165421.

[37] H. Le, N. Pham, A density functional theory investigation of nin, pdn, ptn clusters $(n=1-4)$ adsorbed on buckminsterfullerene $\left(\mathrm{c}_{60}\right)$, ChemPhysChem.

[38] Z.-Y. Tian, S.-L. Dong, Yttrium-dispersed $\mathrm{c}_{60}$ fullerenes as high-capacity hydrogen storage medium, The Journal of chemical physics 140 (8) (2014) 084706 .

[39] L. Genovese, A. Neelov, S. Goedecker, T. Deutsch, S. A. Ghasemi, A. Willand, D. Caliste, O. Zilberberg, M. Rayson, A. Bergman, R. Schneider, Daubechies wavelets as a basis set for density functional pseudopotential calculations, The Journal of Chemical Physics 129 (1).

[40] S. Goedecker, Minima hopping: An efficient search method for the global minimum of the potential energy surface of complex molecular systems, The Journal of Chemical Physics 120 (21) (2004) 9911-9917.

[41] B. Schaefer, S. Alireza Ghasemi, S. Roy, S. Goedecker, Stabilized quasinewton optimization of noisy potential energy surfaces, The Journal of chemical physics 142 (3) (2015) 034112.

[42] S. Goedecker, W. Hellmann, T. Lenosky, Global minimum determination of the born-oppenheimer surface within density functional theory, Physical review letters 95 (5) (2005) 055501.

[43] S. Roy, S. Goedecker, V. Hellmann, Bell-evans-polanyi principle for molecular dynamics trajectories and its implications for global optimization, Phys. Rev. E 77 (2008) 056707.

[44] M. Amsler, J. A. Flores-Livas, L. Lehtovaara, F. Balima, S. A. Ghasemi, D. Machon, S. Pailhes, A. Willand, D. Caliste, S. Botti, et al., Crystal structure of cold compressed graphite, Physical review letters 108 (6) (2012) 065501 . 
[45] M. Amsler, J. A. Flores-Livas, T. D. Huan, S. Botti, M. A. Marques, S. Goedecker, Novel structural motifs in low energy phases of lialh 4, Physical review letters 108 (20) (2012) 205505.

[46] S. Botti, J. A. Flores-Livas, M. Amsler, S. Goedecker, M. A. Marques, Low-energy silicon allotropes with strong absorption in the visible for photovoltaic applications, Physical Review B 86 (12) (2012) 121204.

[47] S. Botti, M. Amsler, J. A. Flores-Livas, P. Ceria, S. Goedecker, M. A. Marques, Carbon structures and defect planes in diamond at high pressure, Physical Review B 88 (1) (2013) 014102.

[48] J. A. Flores-Livas, A. Sanna, E. Gross, High temperature superconductivity in sulfur and selenium hydrides at high pressure, The European Physical Journal B 89 (3) (2016) 1-6.

[49] M. Amsler, J. A. Flores-Livas, M. A. Marques, S. Botti, S. Goedecker, Prediction of a novel monoclinic carbon allotrope, The European Physical Journal B 86 (9) (2013) 1-3.

[50] B. Schaefer, R. Pal, N. S. Khetrapal, M. Amsler, A. Sadeghi, V. Blum, X. C. Zeng, S. Goedecker, L.-S. Wang, Isomerism and structural fluxionality in the au26 and au26-nanoclusters, ACS nano 8 (7) (2014) 7413-7422.

[51] J. A. Flores-Livas, M. Amsler, T. J. Lenosky, L. Lehtovaara, S. Botti, M. A. Marques, S. Goedecker, High-pressure structures of disilane and their superconducting properties, Physical review letters 108 (11) (2012) 117004 .

[52] S. E. Schönborn, S. Goedecker, S. Roy, A. R. Oganov, The performance of minima hopping and evolutionary algorithms for cluster structure prediction, The Journal of chemical physics 130 (14) (2009) 144108.

[53] J. A. Flores-Livas, M. Amsler, C. Heil, A. Sanna, L. Boeri, G. Profeta, C. Wolverton, S. Goedecker, E. Gross, Superconductivity in metastable 
phases of phosphorus-hydride compounds under high pressure, Physical Review B 93 (2) (2016) 020508.

[54] J. P. Perdew, K. Burke, M. Ernzerhof, Generalized gradient approximation made simple, Phys. Rev. Lett. 77 (1996) 3865-3868.

[55] M. A. Marques, M. J. Oliveira, T. Burnus, Libxc: A library of exchange and correlation functionals for density functional theory, Computer Physics Communications 183 (10) (2012) 2272 - 2281.

[56] S. Goedecker, M. Teter, J. Hutter, Separable dual-space gaussian pseudopotentials, Phys. Rev. B 54 (1996) 1703-1710.

[57] A. Willand, Y. O. Kvashnin, L. Genovese, Á. Vázquez-Mayagoitia, A. K. Deb, A. Sadeghi, T. Deutsch, S. Goedecker, Norm-conserving pseudopotentials with chemical accuracy compared to all-electron calculations, The Journal of Chemical Physics 138 (10).

[58] C. Hartwigsen, S. Goedecker, J. Hutter, Relativistic separable dual-space gaussian pseudopotentials from h to rn, Phys. Rev. B 58 (1998) 3641-3662.

[59] K. Lejaeghere, G. Bihlmayer, T. Björkman, P. Blaha, S. Blügel, V. Blum, D. Caliste, I. E. Castelli, S. J. Clark, A. Dal Corso, et al., Reproducibility in density functional theory calculations of solids, Science 351 (6280) (2016) aad3000.

[60] B. Silvi, A. Savin, et al., Classification of chemical bonds based on topological analysis of electron localization functions, Nature 371 (6499) (1994) 683-686.

[61] G. Kresse, J. Furthmüller, Efficient iterative schemes for ab initio totalenergy calculations using a plane-wave basis set, Physical review B 54 (16) (1996) 11169.

[62] G. Henkelman, A. Arnaldsson, H. Jónsson, A fast and robust algorithm for bader decomposition of charge density, Computational Materials Science 36 (3) (2006) 354-360. 
[63] E. Sanville, S. D. Kenny, R. Smith, G. Henkelman, Improved grid-based algorithm for bader charge allocation, Journal of computational chemistry 28 (5) (2007) 899-908.

[64] W. Tang, E. Sanville, G. Henkelman, A grid-based bader analysis algorithm without lattice bias, Journal of Physics: Condensed Matter 21 (8) (2009) 084204 .

[65] F. Rabilloud, Structure and electronic properties of alkali- $\mathrm{c}_{60}$ nanoclusters, The Journal of Physical Chemistry A 114 (26) (2010) 7241-7247.

[66] F. Rabilloud, Electronic and geometric properties of exohedral potassiumfullerenes, Computational and Theoretical Chemistry 964 (1) (2011) 213217.

[67] R. C. Wcast, M. Astle, W. Beyer, CRC handbook of chemistry and physics (1985).

[68] M. Calatayud, J. Andrés, A. Beltrán, B. Silvi, The hierarchy of localization basins: a tool for the understanding of chemical bonding exemplified by the analysis of the vox and $\mathrm{vo}_{x}^{+}(\mathrm{x}=1-4)$ systems, Theoretical Chemistry Accounts 105 (4-5) (2001) 299-308.

[69] A. Savin, B. Silvi, F. Coionna, Topological analysis of the electron localization function applied to delocalized bonds, Canadian journal of chemistry 74 (6) (1996) 1088-1096.

[70] R. Rousseau, D. Marx, Exploring the electronic structure of elemental lithium: from small molecules to nanoclusters, bulk metal, and surfaces, Chemistry-A European Journal 6 (16) (2000) 2982-2993.

[71] Y. Grin, A. Savin, B. Silvi, The elf perspective of chemical bonding, The Chemical Bond: Fundamental Aspects of Chemical Bonding (2014) 345382. 